\title{
Method for Marking and Positioning Corrosions in the Automobile Engine Cylinder Cavity from the Ultrasonic Phased Array C-scan Images
}

\author{
Xiaoxia YANG ${ }^{1, a}$, Bin XUE ${ }^{2, b, *}$, Lecheng $\mathrm{JIA}^{3, c}$, Hao ZHANG ${ }^{3, d}$ \\ ${ }^{1}$ Tianjin Key Laboratory of Information Sensing \& Intelligent Control, Tianjin University of \\ Technology and Education, Tianjin, 300222, China; \\ ${ }^{2}$ School of Marine Science and Technology, Tianjin University, Tianjin, 300072, China \\ ${ }^{3}$ State Key Laboratory of Precision Measurement Technology and Instrument, Tianjin University, \\ Tianjin, 300072, China \\ aemail: yangxiaoxia0925@163.com, bemail: xuebin@tju.edu.cn(Corresponding author), \\ cemail: jialecheng@tju.edu.cn, demail: zhanghao224@tju.edu.cn
}

Keywords: Engine; Corrosion; Ultrasonic phased array; C-scan image

\begin{abstract}
In the automotive remanufacturing movement, the inspection of the corrosion defects on the engine cylinder cavity is a key and difficult problem. In this paper, the ultrasonic phased array is applied to detect the corrosions in this part, and a method for marking and positioning the corrosions from the ultrasonic phased array C-scan images based on a series of image processing algorithms is proposed. Firstly, the image enhancement algorithm is carried out to the C-scan image and the spurious signals are removed; then according to the characteristics of the C-scan image, the improved watershed segmentation algorithm which can avoid over-segmentation, i.e., the morphological labeled watershed algorithm is applied to segment the C-scan image and realize the defect marking; finally, the centroid extraction method is used for the defect positioning work. The final result demonstrates that the method we proposed is effective for marking and positioning corrosions in the automobile engine cylinder cavity from the ultrasonic phased array C-scan images.
\end{abstract}

\section{Introduction}

In recent years, with the rapid development of the automobile industry, there is a large number of scrapped automobiles which cause serious environmental pollution and resource wastes. For this problem, the green remanufacture engineering for the scrapped automobiles has been developed, which can make the discarded parts return to service after a series of testing and processing[1] [2]. The engine is the most important component for an automobile which relates to the driving safety and using performance, so the remanufacturing of the engines is a key issue[3]. The engine cylinder cavity is the space for flowing cooling liquid which is between the engine housing and the cylinder, and with the growth of the service time, the metal material in this part may be corroded, which is likely to cause vehicle failures or even traffic accidents. Therefore, the evaluation for the corrosion defects in the engine cylinder cavity is a key problem, however, it is also a difficult one, because in general, this part cannot be dismantled easily. For this problem, we apply a non-destructive testing method, i.e. the ultrasonic phased array[4] to detect the corrosions in this part. The top view of the engine and the ultrasonic phased array transducer on the working surface is shown in the Fig.1.

The C-scan image obtained by the ultrasonic phased array transducer can help the testers identify the defects visually[5]. However, it is usually inefficient to identify and locate the defects only relying on the artificial vision, and some erroneous judgments may appear because of personnel differences, tiredness, and so on, which cannot satisfy the demand for automated and online detection. Therefore, in this paper, we propose a method for marking and positioning corrosions in the automobile engine cylinder cavity from the ultrasonic phased array C-scan images based on a series of image processing algorithms. 


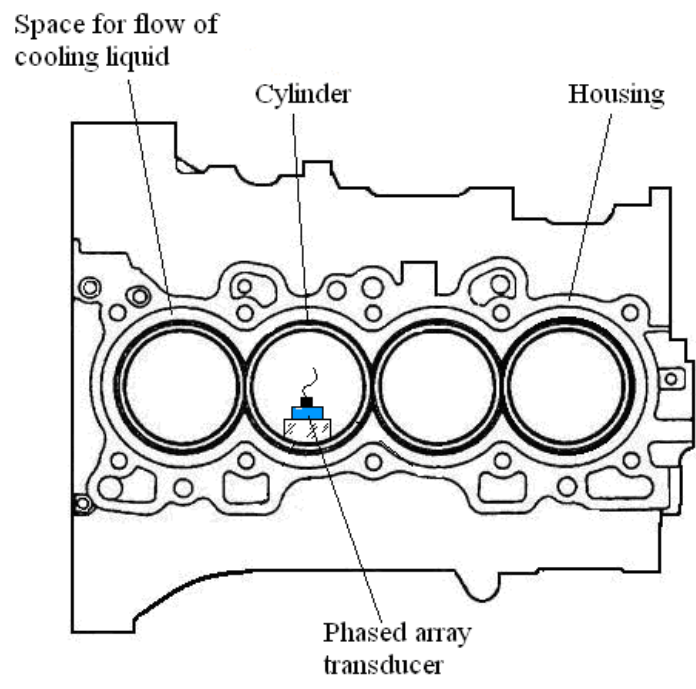

Fig.1. The top view of the engine and the ultrasonic phased array transducer on the working surface

\section{Capture the C-scan images}

In this study, we built an ultrasonic phased array testing system to capture the C-scan images of the corrosion defects, and the main components of this system include a M2M ultrasonic detector, an ultrasonic linear phased array transducer, a wedge with a convex surface installed on the transducer, a PC, and a specimen with some artificial defects. This transducer is 64-element and the width of per element is $0.49 \mathrm{~mm}$, length is $10 \mathrm{~mm}$, the adjacent element interval is $0.1 \mathrm{~mm}$. The Fig.2 shows the whole experimental system.

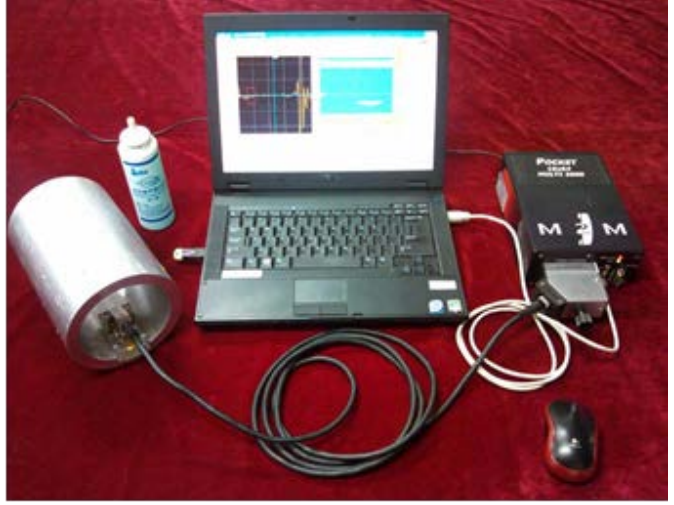

Fig.2. The experimental system

In order to assure the consistency between the signals and the capture positions, an encoder is installed on the transducer to record the signal capture position, which is shown in the Fig.3.

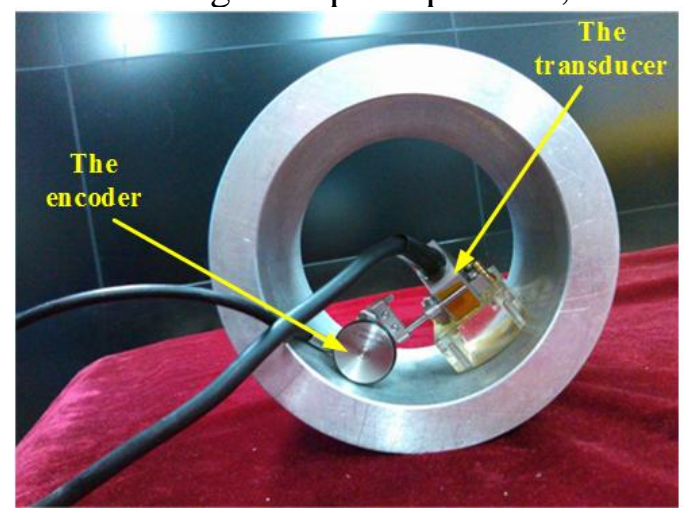

Fig.3. The encoder is installed on the transducer

The Fig. 4 shows the C-scan grayscale image for the artificial defects in the specimen in which six corrosion defects can be observed visually. 


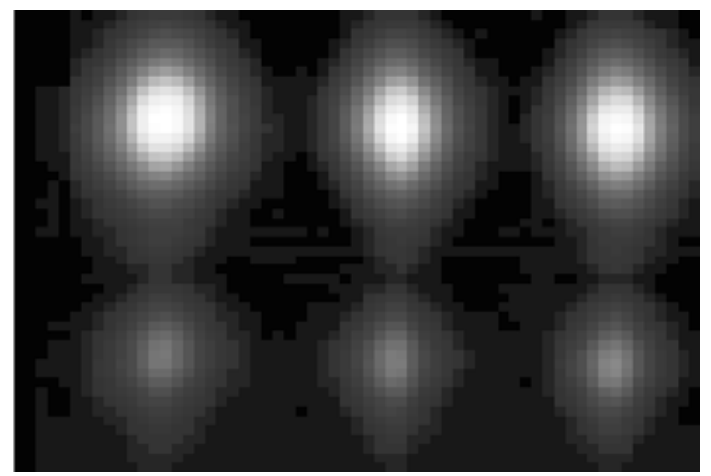

Fig.4. The C-scan grayscale image

\section{Method for marking the corrosions}

To satisfy the demand of automated and online detection for the automobile engine, we propose a method for marking the corrosions from the C-scan images based on a series of image processing algorithms. According to the image-forming principle, one C-scan image may contain a number of defects with different sizes, so it is difficult to find a global threshold to identify them. Besides, it is usually fuzzy around the edge of the corrosions because of the sound beam-width effect. Therefore, in this section, the improved watershed segmentation algorithm which can avoid over-segmentation, i.e., the morphological labeled watershed algorithm is proposed to segment the C-scan image so that realize the automatic making for the defects. In order to obtain a better segmentation result, the enhancement preprocessing is carried out for the image firstly.

\section{C-scan image enhancement}

Observe the image in Fig.4 and we can find that, around the six defects, there are several spurious signals which are caused by the non-uniform thickness of the couplant, multiple reflections of the acoustic wave, etc. In general case, these undesired signals have small amplitudes in a certain range. These spurious signals are useless and may lead to negative effects to the defect identification. So, the purpose of the image enhancement is to eliminate these undesired signals. According to these characteristics, we choose the method of piecewise linear function transformation to enhance the C-scan image.

Set the image grayscale as $L$ and the gray value of the coordinate point $(i, j)$ as $f(i, j)$, then the piecewise linear function is built as followed:

$$
g(i, j)=\left\{\begin{aligned}
0, & f(i, j) \leq 0.03 L \\
f(i, j), & 0.03 L<f(i, j) \leq L
\end{aligned}\right.
$$

where $g(i, j)$ is the gray value of the enhancement image. The capture image is 8-bit, so $\mathrm{L}=255$.

After the enhancement, the original image is transformed as shown in Fig.5 in which the spurious signals are removed.

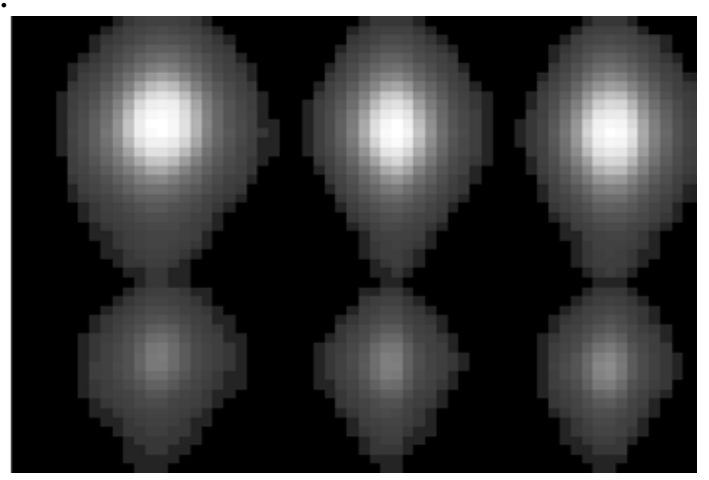

Fig.5. The enhancement image 


\section{C-scan image segmentation}

To mark the defects exactly, in this section, we use the image segmentation technology to mark the defects automatically and get ready for the positioning algorithm in the next section. Analyze the features of the C-scan images and we can find that the edge of each defect is fuzzy and local irregularity is incident because of the influence from the couplant, which is very likely to cause over-segmentation. For this situation, in this paper, the improved watershed segmentation algorithm, i.e., the morphological labeled watershed algorithm is applied to realize the defect marking. The flow chart of this algorithm is shown in the Fig. 6 and the description of the process is as followed:

1) Calculate the gradient of the grayscale image. In this step, the Sobel operator[6] is applied to finish this task.

2) Preprocess the grayscale image, i.e., the morphological opening and closing reconstruction operation[7], which is a kind of nonlinear filter. The most significant advantage of this filter is the ability to keep the boundary information of the images.

3) Label the foreground and the background for the filtered grayscale image in the step 2).

a) Label the foreground. Calculate the local minimal binary image, and carry out the opening and closing operation to smooth edge. Then, remove the local minimal areas whose pixel number less than a certain value and the foreground image is obtained. This foreground image can mark the local minimal value and eliminate the isolated stray elements.

b) Label the background. Calculate the binary image for the filtered grayscale image applying the Otsu method[8], then carry out distance transform and watershed segmentation. The watershed obtained above is the background.

4) Modify the gradient image in the step 1) using the foreground and the background obtained in the step 2). In fact, it is a mandatory modification for the original gradient image in order to remove the undesired local extreme values.

5) Carry out the watershed segmentation operation[9] to the modified gradient image. Then, output the segmentation result and show it in the original grayscale image.

As described above, this algorithm apply the morphological opening and closing reconstruction operation to smooth the grayscale image and eliminate the noise, and the foreground and the background can help to remove the undesired local extreme values. So, the morphological labeled watershed algorithm can effectively prevent the over-segmentation and mark the defects exactly. The marking result by this algorithm is show in the Fig.7 in which we can see that the six defects are marked exactly.

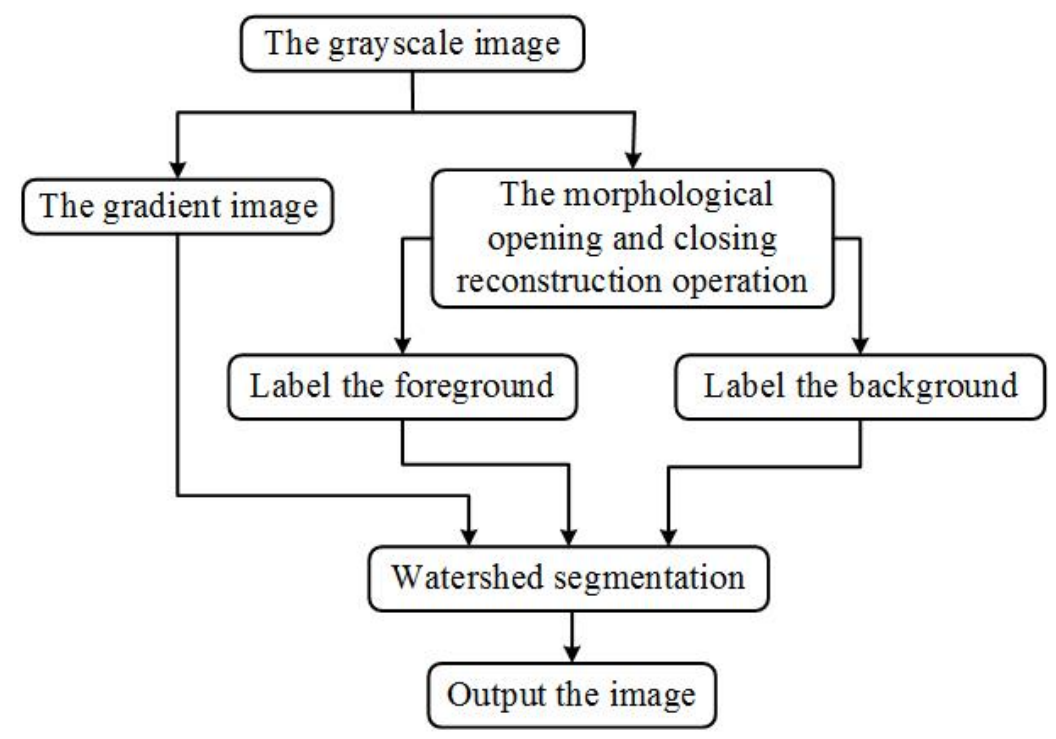

Fig.6. The flow chart of the morphological labeled watershed algorithm 


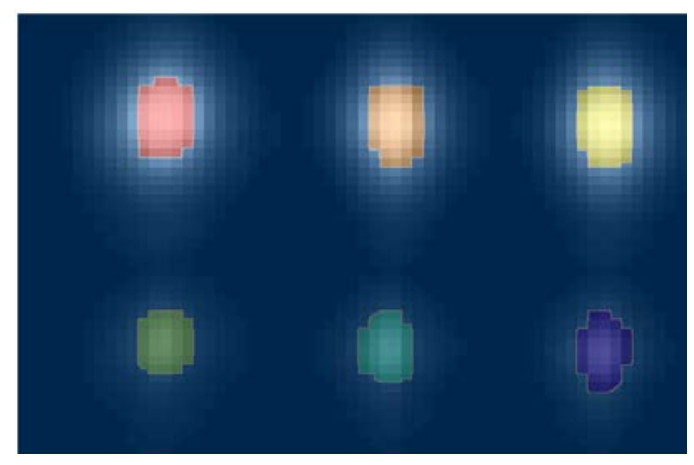

Fig.7. The defect marking result

\section{Method for positioning the corrosions}

The defect positioning is a key task in the testing. In this study, the centroid extraction technology is applied for the positioning work. Set the gray value of the coordinate point $(i, j)$ as $f(i, j)$ in the Fig.7, and the coordinate of the defect centroid noted $\left(x_{m}, y_{m}\right)$ can be expressed as:

$$
x_{m}=\frac{\sum_{i j} i f(i, j)}{\sum_{i j} f(i, j)}, \quad y_{m}=\frac{\sum_{i j} j f(i, j)}{\sum_{i j} f(i, j)}
$$

According the above formula we can see that $x_{m}$ and $y_{m}$ may be non-integer, i.e., the centroid coordinate is sub-pixel accuracy. In other words, the centroid is the expected position of the defect. The defect positioning result for the Fig.7 is shown in the Fig.8, where the star-shaped points express the centroids of the defects. To validate the algorithm, we calculate the distances between two defects and compare to the practical. The five distance values L1-L5 is marked in the Fig.8 and the position result is shown in the table 1. According the data in this table, the mean value of positioning error is $0.33 \mathrm{~mm}$ which can satisfy the measuring requirement for the engine cylinder cavity. The result demonstrates that the method we proposed is effective for marking and positioning corrosions in the automobile engine cylinder cavity from the ultrasonic phased array C-scan images. Number for every mark point and do further analysis towards these defects, such as quantitative analysis, etc., then provide more comprehensive information for the evaluation to the corrosive condition in the engine cylinder cavity.

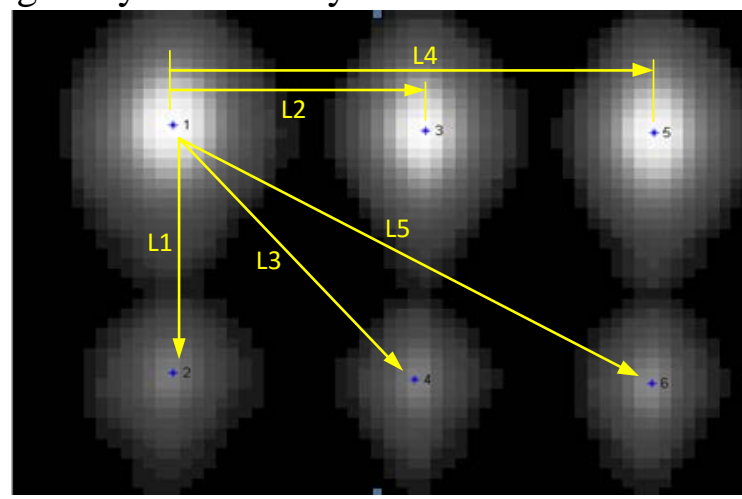

Fig.8. The positioning result for the defects

Table 1 The positioning result $(\mathrm{mm})$

\begin{tabular}{c|ccccc}
\hline & L1 & L2 & L3 & L4 & L5 \\
\hline True value & 15.00 & 19.90 & 24.92 & 39.80 & 42.53 \\
Positioning value & 14.66 & 20.43 & 24.78 & 39.64 & 42.07 \\
Error & 0.34 & 0.53 & 0.14 & 0.16 & 0.46 \\
\hline
\end{tabular}




\section{Conclusions}

The automotive remanufacturing movement can effectively avoid the environment pollution and resource wastes caused by the great number of scrapped automobiles and engines. The evaluation for the corrosion defects in the engine cylinder cavity is a key and difficult problem. In this paper, the ultrasonic phased array is applied to detect the corrosions in this part, and a method for marking and positioning corrosions in the automobile engine cylinder cavity from the ultrasonic phased array C-scan images based on a series of image processing algorithms is proposed. Firstly, the image enhancement algorithm is carried out to the C-scan image and the spurious signals are removed; then the morphological labeled watershed algorithm is applied to segment the C-scan image and realize the defect marking; finally, the centroid extraction method is used for the defect positioning work. The final result demonstrates that the method we proposed is effective for marking and positioning corrosions in the automobile engine cylinder cavity from the ultrasonic phased array C-scan images, which is a preparation for further analysis towards these defects, such as quantitative analysis, etc., and providing more comprehensive information for the evaluation to the corrosive condition in the engine cylinder cavity.

\section{Acknowledgement}

This research is supported by the National Natural Science Foundation of China (61505140) and the projects from Tianjin University of Technology and Education (No.KYQD14049 and No.KJ15-24). The authors would like to express their sincere thanks to them and the comments from the reviewers and the editor would be very much appreciated.

\section{References}

[1] Bingquan Wen,Xia Xie,Bin Wang. Review of Remanufacturing for Automotive Components[C]. In: Applied Mechanics and Materials: Trans Tech Publ, 2012: 482-485

[2] SS Yang,HY Ngiam,SK Ong,et al., The Impact of Automotive Product Remanufacturing on Environmental Performance[J], Procedia CIRP, 2015, 29: 774-779

[3] Xiaoxia Yang,Shili Chen,Fang Sun,et al., Simulation Study on the Acoustic Field from Linear Phased Array Ultrasonic Transducer for Engine Cylinder Testing[J], CMES: Computer Modeling in Engineering \& Sciences, 2013, 90 (6): 487-500

[4] Michael Moles, Ultrasonic Phased Array[J], The NDT Technician, 2012, 11 (3): 1-5

[5] A Chukwujekwu Okafor,Amitabha Dutta. Non-Destructive Evaluation of Concrete with Ultrasonic C-Scan and Digital Image Enhancement Techniques[C]. In: 40th annual review of progress in quantitative nondestructive evaluation: Incorporating the 10th International Conference on Barkhausen Noise and Micromagnetic Testing: AIP Publishing, 2014: 925-933

[6] Haoge Ma,Lu Han, Multi-Technology Integration Based on Low-Contrast Microscopic Image Enhancement[J], Sensors \& Transducers, 2014, 163 (1): 96

[7] Milan Sonka,Vaclav Hlavac,Roger Boyle. Image Processing, Analysis, and Machine Vision[B]. Cengage Learning, 2014

[8] Hetal J Vala,Astha Baxi, A Review on Otsu Image Segmentation Algorithm[J], International Journal of Advanced Research in Computer Engineering \& Technology (IJARCET), 2013, 2 (2): pp: 387-389

[9] Shashi Bala,Amit Doegar, Automatic Detection of Sickle Cell in Red Blood Cell Using Watershed Segmentation[J], Blood, 2015, 4 (6) 